\title{
CORRECTION OF THE LONG-RANGE BEAM-BEAM EFFECT IN LHC USING ELECTRO-MAGNETIC LENSES
}

\author{
J.P. Koutchouk, CERN, Geneva, Switzerland
}

\section{Abstract}

The beams in LHC collide head-on in at most four experimental points. Due to the small bunch spacing, the beams experience more than one hundred 'near-misses' on either side of the collision points. The transverse beam separation at these places, limited by the quadrupole aperture, is in the range of 7 to $13 \sigma$. The non-linear part of these 'longrange' interactions appears to be the dominant mechanism for beam blow-up or beam loss in simulation. A simple non-linear model of the long-range interactions can be devised. It shows that the latter may be locally corrected with good accuracy using wires as correcting lenses. The nonlinearity measured by the tune footprint is reduced by one order of magnitude. Pulsing the correcting lenses cancels the so-called PACMAN effect.

\section{THE LONG-RANGE BEAM-BEAM INTERACTIONS IN LHC}

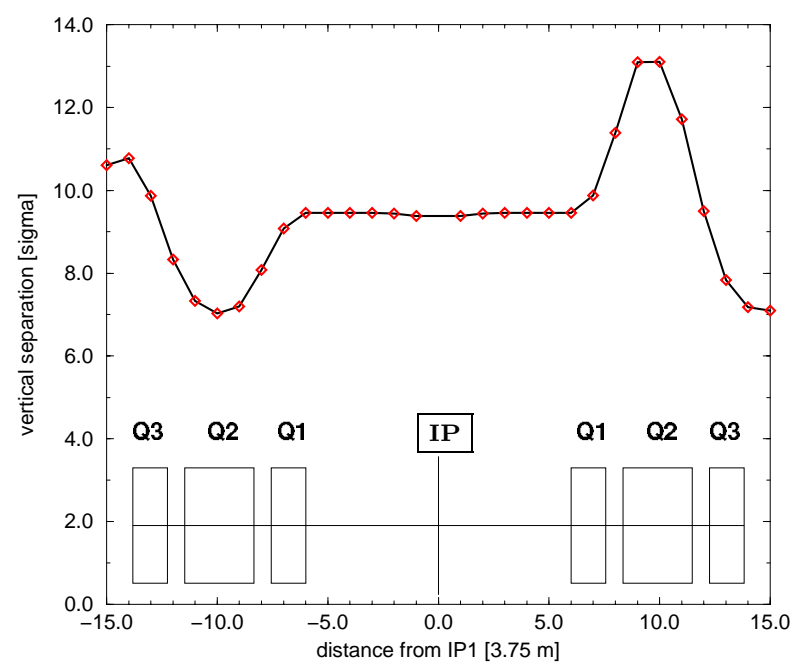

Figure 1: Beam separation in the crossing angle $( \pm 150 \mu \mathrm{rad})$ plane of IP1 in $\sigma$ 's

With a 25 ns bunch spacing, there would be 31 headon collisions per experimental insertion in the absence of the $\pm 150 \mu \mathrm{rad}$ crossing angle. The aperture of the singlebore low- $\beta$ quadrupoles does not allow to increase it much above its nominal value. In the high-luminosity proton mode, the beam size is squeezed in two of the four collision points (IP1 and IP5). The larger beam divergence sets the normalized beam separation to $9.5 \sigma$ on average (Figure 1). In the other two collision points, the normalized separation is much larger. Their contribution to the long-range (LR) beam-beam effect can be neglected.

The machine parameters were chosen to limit to 0.01 the tune spread due to the beam-beam effect. This criterion, successfully tested in the $\mathrm{Sp} \bar{p} \mathrm{~S}$ for the head-on beam-beam effect, is extended, for LHC to its LR component as well. In spite of the crossing angle, the footprint of the latter is still $65 \%$ of that due to the head-on collision.

Tracking studies using as a criterion the dynamic aperture [1] [2], the diffusion in tune or amplitude [4] [3] [5] have demonstrated the importance of the LR interactions. Even-though the footprint criterion is fulfilled, losses of particles occurs at $8.5 \sigma$ and a significant diffusion in amplitude and tune is observed at lower amplitudes. The LR effect acts as the dominant destabilizing mechanism.

The alternating crossing angles [6] in IP1 and IP5 minimize the tune footprint by a compensation of the linear detunings. We propose in this paper a correction principle able to cope with the non-linear part as well.

\section{MODEL OF THE LONG-RANGE BEAM-BEAM KICKS}

We consider a slightly simplified model of the LR beambeam interactions for the design of the correction system. The test of its efficiency is carried out without these simplifications. Only one of the two identical insertions is considered without losing generality. Following the tradition, the sample particle of one beam is called the weak beam. It suffers from the perturbation of the second 'strong' beam.

\subsection{Layout and Strength of the LR Effect}

Due to the strong focusing of the low- $\beta$ quadrupoles, the 15 LR kicks experienced by the weak beam on each side of an IP are very close in betatron phase. Their average and rms phase shifts from the IP are $88.5^{\circ}$ and $2.0^{\circ}$. For $80 \%$ of the kicks, the rms phase difference is $0.4^{\circ}$ only. We can therefore lump the kicks.

The anti-symmetric optics of the low- $\beta$ section causes the Twiss parameters to be unchanged when changing simultaneously the plane and the side of the IP. Figure 1 shows that the beams are round to within $10 \%$ in about $60 \%$ of the cases. The largest aspect ratio is about 1.8 as compared to a beam separation of at least 7 in the same units. We therefore assume in the model round beams.

The nominal number of particles per bunch is $1.110^{11}$ for a rms bunch length of $7.7 \mathrm{~cm}$. Assuming a total bunch length $\sqrt{2 \pi} \sigma_{s}$, the instantaneous beam current is $27.36 \mathrm{~A}$ (43.77 A for the ultimate performance). 


\subsection{Model of the Beam Magnetic Field}

For ultra-relativistic counter-rotating beams, the forces exerted by the electric and magnetic fields are equal. The interaction time is half the bunch length. The kick is the same when integrating the magnetic force only over the full bunch length. Assuming a Gaussian cylindrical charge distribution, the expression of the magnetic field is:

$$
B_{\theta}=\frac{\mu_{0}}{2 \pi} \frac{I_{b}}{r}\left(e^{-r^{2} / 2 \sigma_{S}^{2}}-1\right)
$$

$I_{b}$ is the beam current, $r$ the distance between the center of the strong beam and the weak beam, $\sigma_{S}$ the transverse beam size of the strong beam.

The largest amplitude of the betatron oscillation allowed by the collimation system is $6 \sigma$. In the worst case where this amplitude is fully in the plane of the beam separation, the exponential term in Eq. 1 accounts for $4 \%$ of the integrated magnetic field over the 30 LR interactions. Averaging over the betatron phase, it is reduced to $1 \%$.

An electric current in a wire is therefore a good model for the strong beam in the LHC LR interactions. The multipole expansion of this model magnetic field is given by:

$B_{y}+i B_{x}=\frac{\mu_{0} I_{b}}{2 \pi r_{0}} \sum_{n=1}^{\infty}(-\cos n \phi-i \sin n \phi) \frac{(x+i y)^{n-1}}{r_{0}^{n-1}}$

where $r_{0}$ is the closed orbit difference between the beams, $\phi$ the azimuthal angle and $x$ and $y$ the betatron coordinates of the weak beam. A naive calculation of e.g. the integrated $b_{10}$ shows an integral much larger than that due to the low$\beta$ quadrupole field imperfections and may give a clue to the seriousness of the LR effect.

\subsection{Scaling of the LR Perturbation}

The perturbation of the motion is proportional to:

$$
\int_{l_{L R}} \sqrt{\beta_{x / y}} \frac{B_{y / x}}{B \rho} d s
$$

If $r_{0}, x$ and $y$ scale with the same $\sqrt{\beta}$, the perturbation does not depend on the $\beta$-function. This is the case for about all LR encounters in the experimental straight-section (about $50 \%$ of the total).

\section{LR CORRECTION SCHEME}

\subsection{Principle of the Correction}

The model of the LR beam-beam kicks points clearly to the possibility of correcting the LR beam-beam effect (linear and non-linear) by means of an electric current in a conductor running parallel to the weak beam. Assuming such a corrector on each side of the IP, the integrated intensity shall be $27.36 \mathrm{~A} \times \sqrt{2 \pi} \times 7.7 \mathrm{~cm} \times 15 \approx 80 \mathrm{~A}$ m with a sign opposite of that of the strong beam.
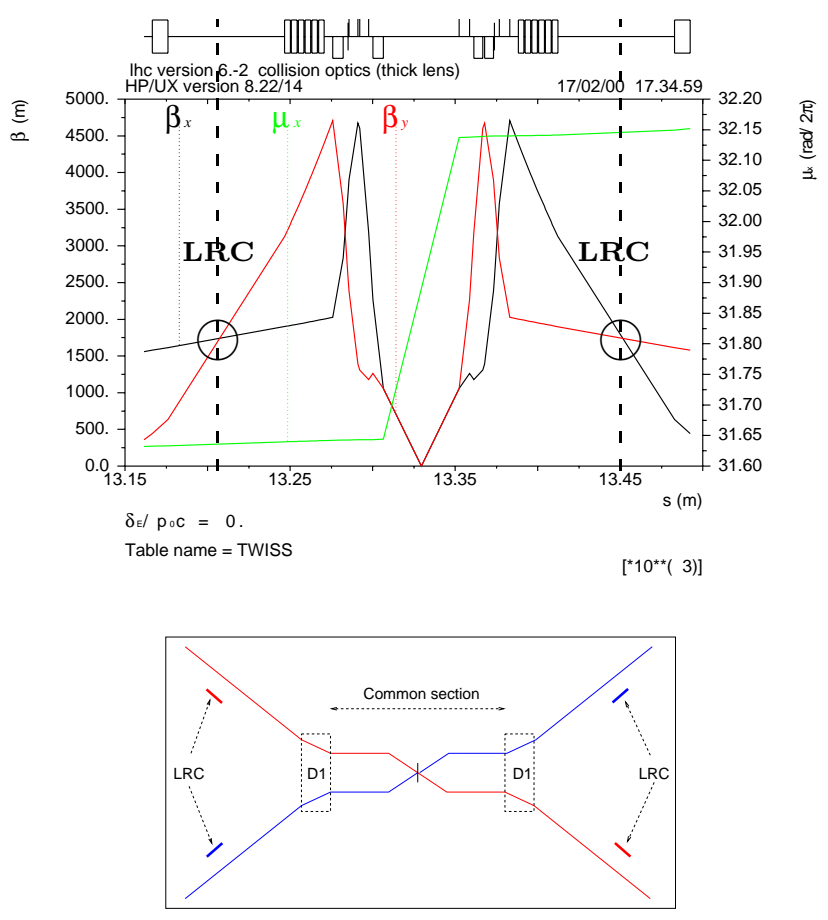

Figure 2: Twiss parameters in IR5 and schematic positions of the correctors for a horizontal crossing.

\subsection{Transverse Position of the Correctors}

The respective transverse positions of the corrector and weak beam should be the same as that of the strong and weak beams at the LR interaction points. For a horizontal crossing, the correctors should be placed between the two beams. For a vertical crossing, they should be placed above or below the weak beam. Eq. 3 remains invariant if the beam-corrector separation $r_{0}$ scales with the $\beta$-function. Hence the correctors shall be placed at $9.5 \sigma$ from the weak beam. This provides an exact correction of the LR interactions occurring in the straight-section and an approximate one for the others. This approximation is later investigated numerically. Eq. 3 shows further that any other scaling, i.e. $I$ versus $r_{0}$ does not allow the simultaneous compensation of all linear and non-linear terms. The separation of $9.5 \sigma$ puts the corrector in the shadow of the secondary collimator. Figure (2) shows a schematics of the corrector set-up.

\subsection{Longitudinal Position of the Correctors}

Positions with equal $\beta$-functions in the two planes may be found on the other side of the triplet versus the IP (Figure 2). At these positions ( $112 \mathrm{~m}$ from the IP's), the beam separation is almost nominal $(\approx 100 \sigma)$ and sufficient to install instrumentation acting on a single beam. The betatron phase shift between these positions and the LR interaction points is $2.6^{\circ}$, owing to the very large $\beta$-functions. This small phase shift should guaranty that the phase-dependent non-linear terms are well compensated (e.g. factors of 5 and 2 for 5 th and 11 th-order resonances). 


\section{NUMERICAL SIMULATIONS}

\subsection{Model for the Simulation}

LHC v6 is used in its full complexity. To allow a faster evaluation in the MAD tracking, the part of the lattice which does not include LR interactions or correctors is mapped at the order 3 in the Hamiltonian. The LR corrector is represented by a beam-beam lens whose beam size is artificially reduced $(\sigma / 100)$, providing a field in $1 / r$ with great accuracy. The correctors are positioned on each side of the IP at a position where the $\beta$-functions are the same in the two planes on the unperturbed machine. They are transversely displaced by $9.5 \sigma$. The criteria used to judge on the efficiency of the correction are: the betatron tunes, the closed orbit at another collision point and the largest extent of the tune footprint. The latter is calculated by tracking a set of initial conditions on circles in the $x, y$ plane with radii ranging from $\sqrt{x^{2}+y^{2}}=1 \sigma$ to $6 \sigma$. By symmetry, there is no orbit perturbation at the IP under consideration.

\subsection{Test of the Correction}

This artificial example allows an exact correction. Only the LR interactions occurring in the experimental straightsection are retained (12 in total) and their strength increased to keep the same integrated kick. Table 1 shows a practically perfect correction.

\begin{tabular}{|l|c|c|c|}
\hline Name & $Q_{x}$ & $Q_{y}$ & $\Delta Q(6 \sigma)$ \\
\hline no beam-beam & .2800 & .3100 & $.003310^{-3}$ \\
\hline 12 LR's & .2820 & .3080 & $2.210^{-3}$ \\
\hline after correction & .2800 & .3100 & $.009210^{-3}$ \\
\hline
\end{tabular}

Table 1: Results of the correction test

\subsection{A Realistic Correction in IP5}

The LR interactions in the quadrupoles are now added. Table 2 and Figure 3 show that the compensation is almost exact for the orbit and reduces the footprint by a factor of 5. If the current in the correctors is empirically increased

\begin{tabular}{|l|c|c|c|c|}
\hline Name & $Q_{x}$ & $Q_{y}$ & $\begin{array}{c}x_{I P 1} \\
\mu \mathrm{m}\end{array}$ & $\begin{array}{c}\Delta Q(6 \sigma) \\
10^{-3}\end{array}$ \\
\hline no beam-beam & .2800 & .3100 & 0 & .0033 \\
\hline All LR's & .2824 & .3076 & 2.6 & 3.5 \\
\hline Nominal correction & .2802 & .3098 & .09 & .65 \\
\hline Optimized correction & .2799 & .3100 & .25 & .275 \\
\hline
\end{tabular}

Table 2: Results of a realistic correction in IP5

by $13 \%$, the footprint is further reduced by a factor 2 and the orbit perturbed in a negligible way. Given the small betatron phase shift between perturbation and correction, the tune footprint should be a rather faithful image of the other non-linear terms.

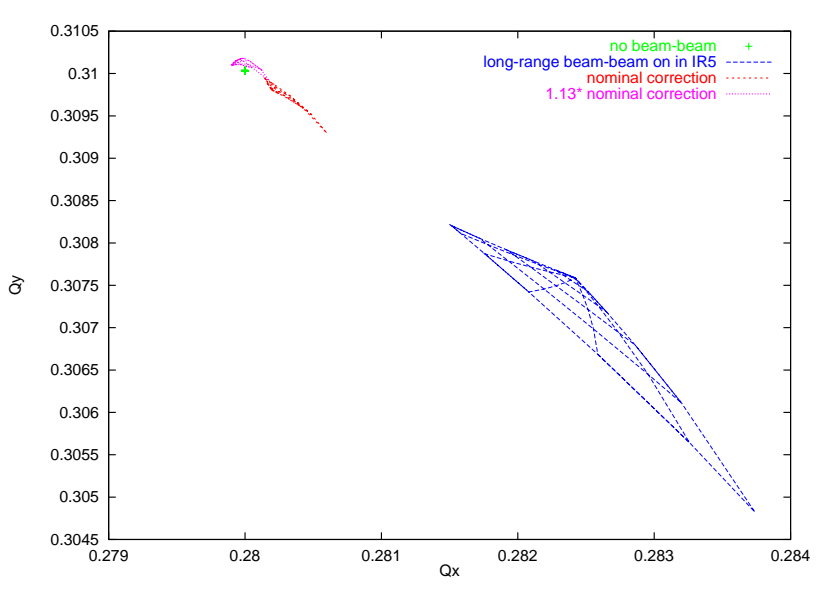

Figure 3: Tune footprints before and after corrections

\subsection{Robustness}

Due to the summation of the LR kick over 15 bunches, the expected $20 \%$ bunch intensity spread is reduced to a $5 \%$ effect. The scheme is insensitive to a change of the closed orbit of the weak beam. The dependence on the exact corrector current appears non critical (Table 2).

\section{CONCLUSION}

We show that the long-range beam-beam interactions, presently considered as the most drastic limitation of LHC performance, can be rather accurately corrected for both their linear and non-linear perturbations. The principle of the corrector is simple and considered challenging but feasible by the experts. A dc operation of the correctors compensates the LR effect for nominal bunches. The so-called PACMAN bunches can be taken care off by pulsing the system at $1 / 15$ of the bunch frequency.

\section{ACKNOWLEDGEMENTS}

To S. Fartoukh, H. Grote, J.M. Jowett and G. Schröder.

\section{REFERENCES}

[1] W. Chou, D. Ritson, 'Dynamic aperture studies during collisions in the LHC', CERN LHC Project Report 123, 1997.

[2] J. Miles in M. Boege et al., 'Overview of the LHC dynamic aperture studies', LHC Project Report 106, 1997.

[3] T. Sen et al., 'Effect of the beam-beam interactions in the LHC', CERN-SL-99-039 AP, 1999.

[4] Y. Papaphilippou, F. Zimmermann, 'Weak-strong beambeam simulations for LHC', CERN-SL-99-039 AP, 1999.

[5] H. Grote et al., 'LHC Dynamic Aperture at Collision', LHC Project Note 197, 1999.

[6] W. Herr and J. Miles, 'Optimizing the LHC Interaction Region for Luminosity', LHC Project Report 40, 1996.

[7] H. Grote, O. Meincke, 'Tune Footprints for Collision Optics 5.0', LHC Project Note 161, 1998. 